\title{
Judgments for inaccessible targets: Comparing recognition without identification and the feeling of knowing
}

\author{
Jason S. Nomi • Anne M. Cleary
}

Published online: 15 June 2012

(C) Psychonomic Society, Inc. 2012

\begin{abstract}
Both the recognition-without-identification (RWI) and feeling-of-knowing (FOK) paradigms attempt to tap participants' sense about a target that is inaccessible. In the case of RWI, participants judge the likelihood that the inaccessible target was studied. In the case of FOKs, participants judge the likelihood that the inaccessible target will be recognized at a later point in time. The present study compared the two using a hybrid paradigm. The patterns of responding differed depending on whether the judgment was of the RWI type or the FOK type. For the former, ratings were significantly higher for studied than for unstudied inaccessible targets (the typical RWI effect). For the latter, ratings were significantly lower for studied than for unstudied inaccessible targets (a reversal of the usual RWI effect). Similarity of the targets and distractors diminished the usual RWI effect, but not its reversal in the FOK situation, suggesting that the bases of the two effects differ. A potential explanation is that when participants judge whether an inaccessible target was presented earlier, they are oriented toward relying on cue familiarity, which should be greater for cues of studied targets, whereas when they judge whether they will recognize an inaccessible target later on, they are oriented toward relying on the accessibility of information, which is greater in the unstudied condition, due to the lack of priming of the targets.
\end{abstract}

Keywords Recognition without identification . Feeling of knowing $\cdot$ Retrieval failure $\cdot$ Metamemory

J. S. Nomi $\cdot$ A. M. Cleary $(\bowtie)$

Department of Psychology, Colorado State University,

1876 Campus Delivery,

Fort Collins, CO 80523-1876, USA

e-mail: Anne.Cleary@colostate.edu
Most people have had the uncanny experience of recognizing a person as familiar while failing to identify the person. Research aimed at understanding such experiences has spanned different domains within cognitive psychology. Two such domains are the study of recognition without identification (RWI) and of feelings of knowing (FOKs). The domains are similar, in that they both examine judgments given in instances in which participants fail to retrieve a target item. However, they do differ in some regards. In the RWI paradigm (e.g., Cleary, 2006), participants judge the likelihood that an inaccessible target was presented earlier in the experiment. In the FOK paradigm (e.g., Hart, 1965), participants judge the likelihood that they will recognize the inaccessible target word if presented with it later. In the former case, the judgment is retrospective, whereas in the latter case, it is prospective. Furthermore, whereas RWI judgments might be considered episodic in nature (i.e., judging whether a name was presented earlier in the experiment), FOK judgments might be considered semantic in nature (i.e., judging whether a name is known in one's knowledge base well enough to be recognized later). Still, it has been suggested that at least some forms of RWI judgments may tap semantic memory mechanisms (Cleary, 2006), such as those specified by the source-of-activation confusion model regarding how familiarity-based recognition may operate (e.g., Reder et al., 2000). Thus, it is possible that RWI and FOK judgments share the same basis. The present study investigated this possibility.

\section{Recognition without identification}

RWI is the finding that, when identification of target items is prevented, participants can discriminate between those that came from an earlier study list and those that did not, as 
when participants discriminate unidentified fragments of studied words from those of unstudied words (Cleary \& Greene, 2000, 2001; Peynircioğlu, 1990). The RWI effect has been shown with many types of stimuli and in many situations (e.g., Cleary, 2006; Cleary, Langley, \& Seiler, 2004; Cleary \& Reyes, 2009; Cleary \& Specker, 2007; Cleary, Winfield, \& Kostic, 2007; Kostic \& Cleary, 2009), and not all forms of RWI are perceptual in nature. Cleary (2006) presented participants with a list of answers to general knowledge questions (e.g., Toto) at study. At test, the participants were presented with a list of general knowledge questions. Half of the questions had had their answers presented at study (e.g., "What was the name of the dog in the Wizard of Oz?"), and half had not. Even when the participants were unable to retrieve an answer in response to a question, they were asked to rate the likelihood that it had been presented during study. Among questions that participants had failed to answer, higher ratings were given to those whose answers had been studied than to those whose answers had not been. Because the answers and the questions were not presented together within the experiment, this particular form of RWI involved preexperimental semantic knowledge (as opposed to experiment-specific information).

Other forms of knowledge-dependent RWI have been shown with pictures and their referents. Cleary and Reyes (2009) had participants study the names of famous locations (e.g., "Taj Mahal") and then tested the participants with pictures of famous places. Half of the test pictures were of scenes whose names had been studied, and half were of scenes whose names had not been studied. Among the scenes that could not be identified, participants gave higher recognition ratings to those whose names had been studied than to those whose names had not. In a similar study, Cleary and Specker (2007) showed the same effect when celebrity names and faces were used.

Interestingly, in these aforementioned cases of knowledgedependent RWI (Cleary, 2006; Cleary \& Reyes, 2009; Cleary \& Specker, 2007), participants were prompted for partial information about each inaccessible target (e.g., the word's first letter, its sound, or its number of syllables). Even when instances of correct partial retrieval were removed from the pool of data under consideration, discrimination between inaccessible studied targets and inaccessible unstudied targets still occurred.

\section{A theoretical framework for RWI}

A theoretical framework that has been useful for explaining the RWI phenomenon is the dual-process approach to recognition memory. According to dual-process theory (e.g., Mandler, 1980, or see Yonelinas, 2002, or Diana, Reder,
Arndt, \& Park, 2006, for reviews), old-new discrimination among recognition test items can be based either on recollection (i.e., retrieval of the specific item or episode from the study list) or familiarity (a general sense that the test item is familiar). From this perspective, RWI may reflect familiarity; in the absence of an ability to identify the target studied item from the test probe, participants may rely on the familiarity of the test probe itself in making their recognition judgments (see the General Discussion for how such familiarity might work).

Though RWI seems to fit well within dual-process theories of recognition memory, the RWI method itself is unique among dual-process methods in that it is the only recognition method that involves judgments for currently inaccessible targets at test. Other existing methods of separating familiarity from recollection generally test recognition of fully identifiable, already-studied items. In this regard, the RWI method can be seen as more similar to the method used to study the FOK phenomenon than it is to typical dualprocess approaches to recognition memory. Busey, Tunnicliff, Loftus, and Loftus (2000) made a similar point about the differences between recognition memory paradigms and FOK paradigms: "A retrospective confidence rating is one obtained at the time of test about how confident the person is that he/she has made the correct recognition decision. In recognition, these confidence ratings differ from feelings of knowing . . . in that they are given for every recognition judgment, not just after recall failures" (p. 27, emphasis in original). In focusing on retrospective judgments given in cases of recall failures, the RWI paradigm presents a means of specifically comparing retrospective judgments for inaccessible targets with prospective judgments for inaccessible targets.

\section{The FOK phenomenon}

In the FOK paradigm, like the RWI paradigm, researchers examine participants' judgments for inaccessible targets, and, as in some forms of the RWI paradigm, the judgments concern preexperimental knowledge. However, whereas in the RWI paradigm the judgments are retrospective in nature, in the FOK paradigm the judgments are prospective in nature. In the first published FOK study, Hart (1965) asked participants to answer general knowledge questions (e.g., "What is the largest planet in the solar system?"). When participants failed to recall the answer, they rated the likelihood that they would be able to recognize the answer in a future forced choice recognition test. Hart compared the participants' predictions with their actual performance on the recognition test, demonstrating that people could predict above chance which currently inaccessible answers would be recognized on the later test. Although the preferred 
method of measurement has changed since Hart's study (Costermans, Lories, \& Ansay, 1992; Wright, 1996), numerous studies have followed Hart's seminal work (e.g., Gruneberg \& Monks, 1974; Izaute, Chambers, \& Larochelle, 2002; Yaniv \& Meyer, 1987) and have produced a sizable literature on the FOK phenomenon (see Koriat, 2007, for a review).

\section{The FOK phenomenon: Existing theory}

Although many theories of the FOK phenomenon have been proposed over the years (e.g., Nelson, Gerler, \& Narens, 1984; Yaniv \& Meyer, 1987), the currently dominant theoretical framework is a two-stage account (Koriat \& Levy-Sadot, 2001). The first of the two stages is cue familiarity, which is akin to the familiarity component of dual-process theories of recognition memory, and research suggests that cue familiarity (i.e., the familiarity of the test probe or test question itself) can serve as a basis for FOKs (e.g., Benjamin, 2005; Metcalfe, Schwartz, \& Joaquim, 1993). The second stage is accessibility (Koriat, 1993, 1995, 2007), whereby participants essentially rely on a form of the availability heuristic (e.g., Tversky \& Kahneman, 1974), attributing any retrieved information, whether correct or incorrect, to the likelihood that they will recognize the target if presented with it later (Koriat, 1993, 1995; Koriat \& Levy-Sadot, 2001). Cue familiarity is likely the first stage, whereby participants first assess the familiarity of the cue; if it is sufficiently familiar, this familiarity prompts them to proceed to the second stage - the accessibility stage.

\section{The RWI and the FOK phenomena: How comparing the two can inform theory}

With both the RWI and FOK phenomena, participants are making judgments, usually in the form of ratings, about targets that are currently inaccessible. Besides the potential episodic/semantic memory difference, a major difference between the RWI and FOK phenomena is the type of judgment (retrospective vs. prospective). ${ }^{1}$ The RWI phenomenon has not existed for as long in the literature; thus, a more extensive theoretical literature exists on the FOK phenomenon. Because so little is known about the RWI phenomenon, it is conceivable that it has the same underlying basis as the FOK phenomenon, particularly given speculation that it

\footnotetext{
${ }^{1}$ There may be some qualitative differences between certain FOK judgments and RWI judgments. Some FOK studies may simply ask participants to judge the likelihood of recognition at any point in the future, not just in this particular experimental session. In the present study, we were careful to hold everything constant except for the type of judgment (retrospective vs. prospective) in order to minimize any additional qualitative differences between tasks.
}

may at times result from semantic memory processes (Cleary, 2006). If so, existing FOK theory may be able to inform theory regarding the RWI phenomenon. For example, it is possible that like the FOK phenomenon, RWI is driven by a two-stage process in which cue familiarity is first assessed, and then a search for accessible information is initiated if the cue is found to be familiar. However, if RWI and FOKs were shown to behave differently in response to the same experimental manipulation, this would help to inform RWI theory as well as FOK theory by helping to establish what may be unique about the RWI phenomenon. If we can establish what distinguishes RWI from FOKs, we may come to have a better understanding of both phenomena.

Although there are many similarities between RWI and FOKs, there is also reason to suspect that they may be different metacognitive phenomena. First, other seemingly similar metacognitive phenomena have been empirically shown to differ. For example, the tip-of-the-tongue phenomenon has previously been viewed as similar enough to the FOK phenomenon to inform theory regarding the latter phenomenon (e.g., Koriat, Levy-Sadot, Edry, \& de Marcas, 2003), yet the two have been dissociated both experimentally (Schwartz, 2008) and neurologically (Maril, Simons, Weaver, \& Schacter, 2005). Second, some studies have shown differences between prospective and retrospective judgments with respect to metacognition (e.g., Busey et al., 2000; Dougherty, Scheck, Nelson, \& Narens, 2005). However, these studies did not examine retrospective judgments specifically in instances of current recall failure. ${ }^{2}$ The RWI paradigm presents a unique opportunity to examine retrospective judgments for currently inaccessible targets.

\section{The present study}

In the present study, we directly compared the RWI and FOK phenomena by holding everything constant except the type of judgment (retrospective vs. prospective) for currently inaccessible targets. We used a modified version of the RWI paradigm used by Cleary and Reyes (2009). As in their study, our primary focus was on judgments given to test scenes that could not be identified, but some of our participants gave RWI-type retrospective judgments to the unidentified scenes (i.e., was this scene's name on the study list?), and some gave FOK-type prospective judgments (i.e.,

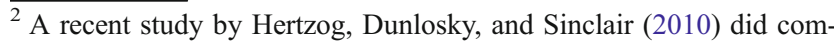
bine FOK judgments with retrospective confidence judgments. However, the retrospective confidence judgments were given during a later forced choice recognition test in which participants were to select the actual studied item from a set of four alternatives, then to rate their confidence in their selection, which is not the same as giving a retrospective judgment to a currently inaccessible target (as the target had been made accessible by the forced choice recognition test).
} 
will you recognize this scene's name if you are presented with it later in the experiment?).

\section{Experiment 1}

The purpose of Experiment 1 was to determine whether the pattern of ratings for pictures of famous scenes that participants could not identify would differ depending on whether participants were rating the likelihood that the inaccessible target scene name had been studied or were rating the likelihood that they would recognize that target scene name if presented with it later.

\section{Method}

Participants A group of 122 introductory psychology students from Colorado State University participated for course credit. These participants were randomly assigned to either the RWI condition or the FOK condition; 61 participated in the RWI condition, and 61 in the FOK condition.

Materials Eighty images of famous scenes and their corresponding names were selected from the stimulus set of Cleary and Reyes (2009) for use in the experiment.

Procedure The participants were told that they would be presented with a list of names of famous locations and that they should try to remember these names for a later memory test. The participants were then presented with a study list of 40 famous scene names (e.g., Sistine Chapel) one at a time for $2 \mathrm{~s}$ each in the upper left-hand corner of the screen, with a 1 -s interstimulus interval.

Following the study list, the test session began. The participants were informed that they would be presented with 80 images of famous locations and that they would be asked to answer several questions for each. First, they would be asked to attempt to name the scene. Then, regardless of whether they succeeded, they would be asked to give a rating using a scale of 1-10. In the RWI condition, this rating would indicate the probability that the scene's name had appeared on the study list $(1=$ definitely not studied, $10=$ definitely studied $)$. In the FOK condition, this rating would indicate the probability that participants would recognize the name if they were to see it later $(1=$ not at all likely to recognize the name, $10=$ very likely to recognize the name). After giving a rating, the participants were given a second chance to identify the scene (in case its name had come to mind during the rating). Finally, the participants were asked to type in any partial information that they could recall about the scene's identity; they were told that the number of syllables in the name, the first letter of the name, or a similar sounding word to the name would all be examples of partial information about the name. Misspelled names that otherwise correctly identified the targets were later classified as "identified." Unidentified scenes for which correct partial information was identified were classified as "partially identified" and were removed from the pool of data under primary consideration (unidentified scenes), but they were also examined as their own class of items in order to investigate partial recollection itself.

\section{Results}

Full-identification rates are reported in Table 1, and partialidentification rates (rates of reporting partial information about the target scene name in the absence of complete identification of the target scene) are reported in Table 2. For fullidentification rates, a $2 \times 2$ Study Status (studied vs. nonstudied) $\times$ Condition (RWI vs. FOK) mixed-model ANOVA revealed a main effect of study status, $F(1,120)=189.67$, $M S E=.01, p<.001, \eta_{\mathrm{p}}{ }^{2}=.61$, such that participants were significantly more likely to identify a test scene when the name had been presented at study than when it had not. No other effects were found $(F<1)$. For partial-identification rates, a $2 \times 2$ Study Status (studied vs. nonstudied) $\times$ Condition (RWI vs. FOK) mixed-model ANOVA revealed a main effect of study status, $F(1,120)=7.58, M S E=.002, p=.007$, $\eta_{\mathrm{p}}{ }^{2}=.06$, such that, unlike the full-identification rates, partialidentification rates were actually higher for items in the unstudied condition than for those in the studied condition. No other effects emerged $(F<1)$. A paired-samples $t$ test performed on partially identified studied and unstudied items in the FOK condition was significant, $t(60)=2.75, S E M=.007$, $p=.008$, such that more items were partially identified in the unstudied condition. This pattern was not significant in the RWI condition $(p=.18)$.

The primary data of interest were the ratings given to unidentified test scenes. These are shown in Table 3 and exclude ratings given in cases in which accurate partial identification occurred. A $2 \times 2$ Study Status (studied vs. nonstudied) $\times$ Condition (RWI vs. FOK) mixed-model ANOVA revealed a Study Status $\times$ Condition interaction, $F(1,120)=27.37, M S E=$ $.40, p<.001, \eta_{\mathrm{p}}^{2}=.19$, such that ratings in the RWI condition were higher in the studied than in the unstudied categories (the

Table 1 Mean scene identification rates (standard deviations in parentheses)

\begin{tabular}{llllll}
\hline & \multicolumn{2}{l}{ RWI Condition } & & \multicolumn{2}{l}{ FOK Condition } \\
\cline { 2 - 3 } \cline { 5 - 6 } & $\begin{array}{l}\text { Name } \\
\text { Studied }\end{array}$ & $\begin{array}{l}\text { Name } \\
\text { Nonstudied }\end{array}$ & & $\begin{array}{l}\text { Name } \\
\text { Studied }\end{array}$ & \multicolumn{1}{l}{ Name } \\
& Nonstudied \\
\hline Experiment 1 & $.49(.15)$ & $.34(.14)$ & & $.51(.15)$ & $.35(.14)$ \\
Experiment 2a & $.44(.18)$ & $.29(.15)$ & & $.40(.14)$ & $.24(.12)$ \\
Experiment 2b & $.41(.16)$ & $.28(.14)$ & & $.40(.15)$ & $.30(.12)$ \\
\hline
\end{tabular}


Table 2 Mean partial-identification rates (standard deviations in parentheses)

\begin{tabular}{llllll}
\hline & \multicolumn{2}{l}{ RWI Condition } & & \multicolumn{2}{l}{ FOK Condition } \\
\cline { 2 - 3 } \cline { 5 - 6 } & $\begin{array}{l}\text { Name } \\
\text { Studied }\end{array}$ & $\begin{array}{l}\text { Name } \\
\text { Nonstudied }\end{array}$ & & $\begin{array}{l}\text { Name } \\
\text { Studied }\end{array}$ & \multicolumn{1}{l}{ Name } \\
& Nonstudied \\
\hline Experiment 1 & $.05(.05)$ & $.06(.05)$ & & $.05(.04)$ & $.07(.05)$ \\
Experiment 2a & $.08(.07)$ & $.09(.06)$ & & $.08(.05)$ & $.11(.06)$ \\
Experiment 2b & $.05(.04)$ & $.07(.05)$ & & $.07(.06)$ & $.08(.06)$ \\
\hline
\end{tabular}

typical RWI effect), whereas ratings in the FOK condition showed the reverse pattern: Ratings in this condition were higher for unstudied than for studied items. A paired-samples $t$ test comparing ratings in the studied and unstudied categories in the RWI condition was significant, $t(60)=5.50, S E M=.09$, $p<.001$, demonstrating that the usual RWI effect (e.g., Cleary $\&$ Reyes, 2009) was indeed present. A paired-samples $t$ test comparing ratings in the studied and unstudied categories of the FOK condition was also significant, $t(60)=2.55, S E M=.13$, $p<.05$, suggesting that the reversal of the RWI pattern that was shown in the FOK condition was also in fact significant. This reversal pattern held up in items analyses, as well (when items were treated as participants): In the RWI condition, ratings were higher for inaccessible studied targets $(M=5.26, S D=1.31)$ than for inaccessible unstudied targets $(M=4.77, S D=1.32)$, $t_{\text {items }}(78)=2.73, S E=.18, p<.01$, whereas in the FOK condition, ratings were lower for inaccessible studied targets $(M=4.79, S D=1.83)$ than for inaccessible unstudied targets $(M=5.14, S D=1.69), t_{\text {items }}(76)=2.00, S E=.18, p<.05$. The interaction was present when items were treated as participants as well: $F_{\text {items }}(1,154)=11.22, M S E=1.26, p=.001$.

Overall, the results of Experiment 1 suggest that it makes a difference whether the judgment given to an unidentified test scene about its unretrieved name is about the name's study status or about its future remembrance. Whereas the usual RWI effect (e.g., Cleary \& Reyes, 2009) was found when the judgment was retrospective, the reverse of that pattern was found when the judgment was prospective. Although the reverse pattern in the FOK condition may seem counterintuitive, overall, the pattern suggests that RWI is a distinct phenomenon from the FOK phenomenon.

Table 3 Mean ratings given to unidentified test scenes (standard deviations in parentheses)

\begin{tabular}{|c|c|c|c|c|}
\hline & \multicolumn{2}{|c|}{ RWI Condition } & \multicolumn{2}{|c|}{ FOK Condition } \\
\hline & $\begin{array}{l}\text { Name } \\
\text { Studied }\end{array}$ & $\begin{array}{l}\text { Name } \\
\text { Nonstudied }\end{array}$ & $\begin{array}{l}\text { Name } \\
\text { Studied }\end{array}$ & $\begin{array}{l}\text { Name } \\
\text { Nonstudied }\end{array}$ \\
\hline Experiment 1 & $5.05(1.07)$ & $4.55(1.06)$ & $4.48(1.38)$ & $4.81(1.36)$ \\
\hline Experiment $2 \mathrm{a}$ & $4.43(1.44)$ & $4.27(1.32)$ & $4.36(1.60)$ & $4.81(1.49)$ \\
\hline Experiment $2 b$ & $4.74(0.97)$ & $4.72(1.08)$ & $4.80(1.61)$ & $5.31(1.46)$ \\
\hline
\end{tabular}

It also presents the first case in which the RWI phenomenon has been shown to be experimentally reversible. ${ }^{3}$

Experiments $2 \mathrm{a}$ and $2 \mathrm{~b}$

Our next question concerned how the standard RWI effect might differ from its reversal in the FOK condition. As mentioned, the standard RWI effect is usually interpreted as reflecting the familiarity component of dual-process theory (see the General Discussion for a detailed description of how cue familiarity in the present paradigm might operate). One theoretical explanation for the reversal pattern shown in Experiment 1 is that participants were using cue familiarity in both cases (i.e., for both RWI-type and FOK-type judgments), they were just influenced by the context of the decision with regard to how they used the familiarity.

In the case of RWI judgments, participants might have assumed that increased cue familiarity would indicate a high likelihood of past occurrence within the experiment, whereas in the case of FOK judgments, they may have assumed that increased cue familiarity indicated a lowered likelihood of being able to recognize the target item later on. This might occur because, if the picture itself seemed familiar, the participants might have believed that they should be able to access its name, and if they could not, the scene's familiarity combined with the inability to access its name might have indicated a lowered likelihood of recognizing the name later relative to when the scene itself was unfamiliar. This might have especially been so if participants recognized that increased scene familiarity on the test can be indicative of an increased likelihood that the scene's name has been studied, as the participants might have assumed that if the name had likely been studied and they still could not access it in response to the picture, the name might be particularly difficult to recognize if they were presented with it later on. If this differential use of cue familiarity was the explanation for the RWI reversal pattern shown in Experiment 1, a manipulation that diminished the utility of cue familiarity in discriminating scenes whose names had been studied from scenes whose names had not should reduce both the normal RWI effect and the RWI reversal effect shown in the FOK condition. This was examined in Experiments $2 \mathrm{a}$ and $2 \mathrm{~b}$.

One factor that is likely to affect familiarity-based oldnew discriminability is interstimulus similarity, or the extent to which targets and distractors resemble one another (e.g., Cleary, Morris, \& Langley, 2007). Highly similar targets and distractors are more difficult to discriminate on the basis of

\footnotetext{
${ }^{3}$ Given the usual interest, within FOK studies, in how well judgments predict later memory, some may wonder how well the ratings (RWI or FOK) predicted final memory performance. Although we did include a final recognition test in one of the experiments reported here (Exp. 2b), our experimental paradigm was not conducive to examining gamma correlations, as some of our categories contained few items.
} 
familiarity, because they both tend to be familiar from the study list presentation. Thus, the RWI effect would be expected to diminish in a situation in which the distractors resembled the targets. If the RWI reversal effect in the FOK condition resulted from the same cue familiarity presumed to drive RWI, then this reversal effect, too, should diminish when the distractors resembled the targets.

Another reason why it is important to examine whether the RWI reversal effect in the FOK condition would occur in a situation of high interstimulus similarity among the targets and distractors is that such similarity is common in standard FOK paradigms. Specifically, in order that participants cannot easily guess the correct item on the final recognition test in a standard FOK paradigm, plausible distractors are typically used to ensure that targets or distractors cannot be selected on the basis of their plausibility or implausibility (e.g., Hart, 1965; Schacter, 1983; Shimamura, Landwehr, \& Nelson, 1981; Shimamura \& Squire, 1986). For example, if a picture of the "Great Wall of China" was presented on the final recognition test and a possible foil name for that picture was "Liberty Bell," participants might correctly reject the foil on the basis that the description "bell" has nothing to do with a picture of a wall. However, in an RWI paradigm, plausible distractors might be expected to reduce old-new discriminability by creating interstimulus similarity (e.g., Cleary, Morris, et al. 2007), thereby diminishing the RWI effect.

In Experiments $2 \mathrm{a}$ and $2 \mathrm{~b}$, we examined whether the use of plausible distractors in a situation of high interstimulus similarity would diminish both the standard RWI effect and the RWI reversal effect shown in the FOK situation of Experiment 1. If both effects were diminished, it would support the idea that cue familiarity was being used in both cases, just differently, depending on the context. However, if interstimulus similarity only diminished the RWI effect and not the reversal effect shown in the FOK situation, this would suggest that the reversal pattern in the FOK situation may be driven by something other than test cue familiarity. Furthermore, such persistence of the reversal effect would suggest that this effect is fairly robust within FOK situations, extending even to more standard FOK testing situations.

\section{Method}

Participants In Experiment 2a, 96 Colorado State introductory psychology students participated for course credit: 48 were randomly assigned to the RWI condition, and 48 to the FOK condition. In Experiment 2b, 72 students participated, 35 in the RWI condition and 37 in the FOK condition. ${ }^{4}$

\footnotetext{
${ }^{4}$ Two more participants were run in the FOK condition than in the RWI condition due to a research assistant error regarding which version of the experiment to run.
}

Materials For Experiment 2a, two new sets of stimuli were created. Forty names and their pictures constituted one set of items, and 40 plausible alternatives to this first set constituted the second set. For example, a scene name and its corresponding picture from the first set of 40 was the "Great Wall of China," and its plausible alternative in the second set was "Berlin Wall." For a given participant, one pool of 40 names and the corresponding images were assigned to the studied condition, and the second pool of plausible alternatives for those items was assigned to the nonstudied condition. For counterbalancing purposes, one set of 40 namescene pairs was assigned to the "studied" category for half of the participants, and the other set of 40 was assigned to the "studied" category for the other half, in both the RWI and FOK conditions. All 80 scenes were presented on the scene test (40 whose names were studied and 40 plausible alternatives whose names were not studied).

Experiment 2b used name-scene pairs from Cleary and Reyes (2009) to ensure that the same pattern from Experiment 2 a would be found with these stimuli as well, and thus would not be attributable to idiosyncratic differences between stimulus sets. Eighty scene name-picture pairs were used, 77 of which were taken from the pool of stimuli used by Cleary and Reyes. These 77 items were chosen for their conduciveness to serving as both targets and plausible distractors across participants. Because only 77 of the scenes from Cleary and Reyes met this criterion, three additional scenes from Experiment $2 \mathrm{a}$ were also used to bring the total to 80 name-picture pairs. Similar to Experiment 2a (and different from Cleary \& Reyes, 2009), the "nonstudied" category consisted of items that were similar, plausible alternatives to the items in the studied category, thus creating interstimulus similarity among the targets and distractors.

Design and procedure The procedure for both Experiments $2 \mathrm{a}$ and $2 \mathrm{~b}$ was the same as in Experiment 1.

\section{Results}

As before, the full- and partial-identification rates among studied and unstudied items in the RWI and FOK conditions (Tables 1 and 2, respectively) will be discussed first. In Experiment $2 \mathrm{a}$, for full-identification rates, a $2 \times 2$ Study Status (studied vs. nonstudied) $\times$ Condition $($ RWI vs. FOK) mixed-model ANOVA revealed a main effect of study status, $F(1,94)=247.97, M S E=.005, p<.001, \eta_{\mathrm{p}}{ }^{2}=.73$, such that identification rates were higher overall for scenes whose names had been studied than for scenes whose names had not. No other significant effects resulted from this analysis $(F<1)$. For partial-identification rates (Table 2 ), a $2 \times 2$ Study Status (studied vs. nonstudied) $\times$ Condition (RWI vs. FOK) mixed-model ANOVA revealed a main effect of study 
status, $F(1,94)=11.28, M S E=.002, p=.001, \eta_{\mathrm{p}}{ }^{2}=.11$, such that, as in Experiment 1, the participants were more likely to provide correct partial information for scenes in the unstudied category. A significant Study Status $\times$ Condition interaction was also found, $F(1,94)=4.13, M S E=.002$, $p<.05, \eta_{\mathrm{p}}{ }^{2}=.04$, such that participants were more likely to provide correct partial information for scenes whose names were unstudied in the FOK condition than in the RWI condition.

In Experiment $2 b$, the $2 \times 2$ Study Status (studied vs. nonstudied) $\times$ Condition (RWI vs. FOK) mixed-model ANOVA performed on full-identification rates again revealed a main effect of study status, $F(1,70)=79.43, M S E=.006$, $p<.001, \eta_{\mathrm{p}}{ }^{2}=.53$, such that identification rates were higher overall for test images whose names had been studied than for test images whose names had not. No other significant effects emerged from this analysis $(F=1.0)$. For partial-identification rates, a $2 \times 2$ Study Status (studied vs. nonstudied) $\times$ Condition (RWI vs. FOK) mixed-model ANOVA revealed a main effect of study status, $F(1,70)=4.22, M S E=.001, p<.05, \eta_{\mathrm{p}}{ }^{2}$ $=.06$, such that participants were again more likely to provide correct partial information in the nonstudied than in the studied category. No other significant effects emerged from this analysis $(F<1)$.

The data of primary interest were the ratings given to those test scenes that could not be identified (Table 3). In Experiment 2 a, a $2 \times 2$ Study Status (studied vs. nonstudied) $\times$ Condition (RWI vs. FOK) mixed-model ANOVA revealed a significant Study Status $\times$ Condition interaction, $F(1,94)=8.12, M S E=$ $.54, p<.01, \eta_{\mathrm{p}}{ }^{2}=.08$, such that, as in Experiment 1, the ratings pattern shown in the RWI condition differed significantly from that shown in the FOK condition. No other significant effects emerged from this analysis $(F<2)$. Although a reversal of the RWI pattern was again shown in the FOK condition, $t(47)=$ 2.88, SEM = .16, $p=.006$, no significant RWI effect was shown in Experiment 2a. This suggests that though the FOK pattern remained the same as in Experiment 1, the RWI effect was diminished to the point of nondetection by the use of highly similar items in the studied and nonstudied categories.

To further examine the diminishment of the RWI effect with increased interstimulus similarity, we compared the magnitude of the RWI effect found in Experiment 2a with that found in Experiment 1. A $2 \times 2$ Study Status (studied vs. nonstudied) $\times$ Experiment ( 1 vs. 2$)$ mixed-model ANOVA performed on ratings given to the unidentified scenes in the RWI conditions revealed a significant Study Status $\times$ Experiment interaction, $F(1,107)=4.59, M S E=$ $.36, p<.05, \eta_{\mathrm{p}}{ }^{2}=.04$. This significant interaction suggests that the RWI effect was indeed significantly smaller in magnitude in Experiment 2 than in Experiment 1, which in turn suggests that interstimulus similarity indeed reduces the RWI effect. The same was not true of the FOK reversal effect. The same analysis performed on the ratings given to unidentified scenes in the FOK conditions revealed no interaction $(F<1)$. Together, these results suggest that interstimulus similarity affected the RWI effect but did not affect the FOK reversal effect.

In Experiment 2b, a $2 \times 2$ Study Status (studied vs. nonstudied $\times$ Condition $($ RWI vs. FOK) mixed-model ANOVA revealed a Study Status $\times$ Condition interaction, $F(1,70)=$ 7.37, MSE $=.34, p=.01, \eta_{\mathrm{p}}{ }^{2}=.10$, such that, as in Experiments 1 and $2 \mathrm{a}$, the rating pattern in the RWI condition differed significantly from that shown in the FOK condition. A main effect of study status also emerged, $F(1,70)=6.56$, $M S E=.34, p=.01, \eta_{\mathrm{p}}{ }^{2}=.09$, but, as can be seen in Table 3, this main effect was carried by the ratings in the unstudied FOK condition, as the RWI condition did not show the reversal pattern shown in the FOK condition. More so than in Experiment 2a, despite continuing to differ significantly from the ratings pattern shown in the FOK condition, the RWI condition revealed that the RWI effect itself had disappeared; a paired-samples $t$ test revealed no difference between the ratings given in the studied category and ratings given in the nonstudied category $(p=.90)$. In the FOK condition, a pairedsamples $t$ test revealed a significant difference between the ratings given in the studied category and those given in the unstudied category, $t(36)=3.45, S E M=.15, p=.001$; as in Experiments 1 and 2a, the ratings were higher for unidentified test scenes whose names had not been studied than for unidentified test scenes whose names had been studied.

As in Experiment 2a, the magnitude of the RWI effect differed significantly from that found in Experiment 1. A $2 \times$ 2 Study Status (studied vs. nonstudied) $\times$ Experiment (1 vs. 2b) mixed-model ANOVA performed on ratings given to unidentified scenes in the RWI conditions revealed a significant Study Status $\times$ Experiment interaction, $F(1,94)=10.22, M S E=.26$, $p=.002, \eta_{\mathrm{p}}{ }^{2}=.10$. This significant interaction suggests that the RWI effect was again significantly smaller in magnitude than in Experiment 1, which in turn further suggests that interstimulus similarity indeed reduces the RWI effect. As in Experiment 2a, the same was not true of the FOK reversal effect. The same analysis performed on the ratings given to unidentified scenes in the FOK conditions revealed no interaction $\left[F(1,93)=1.10, M S E=.49, p=.30, \eta_{\mathrm{p}}{ }^{2}=.01\right]$. Together, these results suggest that interstimulus similarity affected the RWI effect but not the FOK reversal effect, and that this pattern was replicable and robust across experiments with different stimuli.

General discussion

\section{Overview of the present study}

Conceptually, recognition without identification (RWI) and the feeling of knowing (FOK) are very similar. In both cases, participants provide judgments about momentarily 
unretrievable targets, and in both cases, the common realworld example for illustrating the phenomenon is that of recognizing a person's face without being able to retrieve such specifics as the person's name (Cleary \& Specker, 2007; Koriat, 1995). One difference between RWI and FOK judgments is that in the former case, the judgment given to the inaccessible target is retrospective (Was the target presented earlier in the experiment?), whereas in the latter case, the judgment given is prospective (Will you recognize the target if presented with it later in the experiment?). Another potential difference is that RWI judgments might be considered as drawing upon episodic memory, whereas FOK judgments might be considered as drawing upon semantic memory (though see Cleary, 2006, for the idea that RWI judgments might sometimes tap semantic memory processes).

Although some studies have suggested that retrospective and prospective judgments differ metacognitively (Busey et al., 2000; Dougherty et al., 2005), these studies did not specifically compare judgments given to currently inaccessible targets. Because the RWI paradigm presents a means of examining retrospective judgments given when the targets are currently inaccessible, the present study compared RWI and FOK judgments in a situation in which everything else was held constant but the type of judgment (prior list occurrence vs. future recognition) for inaccessible targets. In a variation of the scene RWI paradigm used by Cleary and Reyes (2009), we demonstrated that RWI and the feeling of knowing (FOK) differ from one another. Across three experiments, RWI and FOK judgments responded differently to the target's study status. RWI-type judgments led to the standard RWI effect of higher ratings for studied than for nonstudied unretrievable targets in Experiment 1, and this RWI effect diminished to the point of no detectable difference for studied and nonstudied targets when these items were highly similar in Experiments $2 \mathrm{a}$ and $2 \mathrm{~b}$. In contrast, FOK judgments consistently led to a reversal of the standard RWI-type pattern: In all three experiments, FOK judgments led to higher ratings for nonstudied than for studied unretrievable targets, and this reversal effect was unaffected by the high similarity of the studied and nonstudied targets in Experiments $2 \mathrm{a}$ and $2 \mathrm{~b}$.

\section{A cue familiarity account of the standard RWI pattern}

When the judgment concerns whether or not the unretrievable target was studied (as in the RWI condition), participants may assume that increased cue familiarity is indicative of the likelihood that the inaccessible target was presented recently. Indeed, this is the logic behind dual-process theories of recognition memory, which assume that decisions in recognition memory paradigms can be familiarity-based (e.g., Diana et al., 2006; Jacoby \& Whitehouse, 1989;
Mandler, 2008; Yonelinas, 2002); test items that were familiarized through prior presentation in the experiment seem more familiar at test than test items that were not presented earlier in the experiment, and participants use this test item familiarity to discriminate old from new items. Metcalfe et al. (1993) demonstrated that familiarization of test cues through prior presentation also influences participants' judgments for targets that cannot be retrieved (the cue familiarity component of FOK judgments).

A critical difference between how the cues were familiarized in Metcalfe et al.'s (1993) study and ours is that, whereas Metcalfe et al. (as in standard dual-process approaches to recognition) familiarized the cues through prior presentation of the cues themselves, in our study, cue image familiarization would have occurred through prior presentation of the name of the image. Thus, we would have to assume that because of the prior presentation of the image names at study, images of studied names would be more familiar, on average, than images of nonstudied names, and participants may have used this familiarity difference as a basis for their retrospective rating judgments in the RWI condition.

How might increased image familiarity result from the mere presentation of its name when that name cannot be accessed in response to the image? Ryals and Cleary (2012) suggested that cue familiarity when target retrieval fails (e.g., Cleary, 2004; Cleary, Ryals, \& Nomi, 2009) can be brought on by the type of feature-matching process described by global-matching models (e.g., Clark \& Gronlund, 1996). Ryals and Cleary's cues were nonwords (e.g., foneheed) that potentially resembled studied words (e.g., forehead) graphemically. The researchers found that in order for studied features to have an effect on cue recognition ratings given in the absence of target retrieval, the features had to be present in the cue itself. For example, study word concreteness and study word emotionality affected study word retrieval itself, but not cue recognition ratings given when retrieval failed. In contrast, increasing the degree of global match between the features in the test cues and those in the studied items led to increased cue familiarity ratings.

With regard to the present study, it is possible that cue familiarity was brought on by an overlap of semantic features between the images and the studied image names. In theory, images should activate the same subsets of semantic features that their names do (see, e.g., Cree, McNorgan, \& McRae, 2006; Cree \& McRae, 2003; Mitchell et al., 2008). Thus, in the present study, increased cue familiarity for images whose names were studied but could not be retrieved may have been driven largely by semantic feature overlap between the test images and their studied names. As described by global-matching models (e.g., Clark \& Gronlund, 1996), cues whose features have a high degree of match with studied features stored in memory should elicit a greater familiarity signal than do cues whose features have a low 
degree of match with studied features stored in memory. Thus, cue images whose names were studied should be more familiar, on average, than cue images whose names were not studied, even when retrieval of the target name fails.

\section{Ruling out a cue familiarity account of the RWI reversal pattern in the FOK condition}

One explanation for the RWI reversal pattern in the FOK condition is that participants in that condition relied on the same test cue familiarity presumably used to discriminate studied from unstudied targets in the RWI paradigm; however, the context of the FOK condition caused them to use this familiarity differently. Whereas cue familiarity in the RWI condition may have been seen as indicative of an unretrievable target's likely study status, the same cue familiarity in the FOK condition may have been seen as indicative of a lowered likelihood of being able to recognize that unretrievable target later on. If participants believe that the more familiar cues indicate an increased likelihood of their corresponding targets having been studied, they may then infer that if the target was studied and they still cannot retrieve it, the target may be particularly difficult to recognize later.

However, the results of Experiments $2 \mathrm{a}$ and $2 \mathrm{~b}$ help to rule out this cue familiarity account of the RWI reversal pattern in the FOK condition. The fact that similarity of the targets and distractors only diminished the RWI effect and not the reversal pattern shown in the FOK condition suggests that the two judgments were not based on the same cue familiarity, as the diagnosticity of cue familiarity for differentiating studied from unstudied targets should have been equally low in the RWI and FOK conditions when interstimulus similarity was high. Yet, only the RWI effect diminished from such similarity. That the RWI effect diminished due to the similarity of targets and distractors supports the cue familiarity explanation of the RWI effect itself, leaving as the primary question why the RWI effect reverses in the FOK condition.

\section{A theoretical account of the RWI reversal pattern in the FOK condition}

Given that we have ruled out differential use of cue familiarity as an explanation for the RWI reversal pattern in the FOK situation, what then is the reason for reversal of the ratings? The fact that RWI and FOK ratings responded differently to high target-distractor similarity suggests that they have different bases. Additional support for this idea can be seen in the standard deviations reported in Table 3; they are consistently higher for ratings given to unretrieved targets in the FOK condition than for those given to unretrieved targets in the RWI condition.

One possible explanation for the full pattern of results can be found in current FOK theory. According to Koriat and Levy-Sadot (2001), FOKs can be based on either cue familiarity (e.g., Metcalfe et al., 1993; Reder \& Ritter, 1992; Schwartz \& Metcalfe, 1992) or the accessibility of available information (Koriat, 1993, 1995). It is possible that the RWI and FOK conditions encouraged differential reliance on these two stages (cue familiarity vs. accessibility). Specifically, the RWI condition may have encouraged reliance on cue familiarity, whereas the FOK condition may have encouraged reliance on accessibility.

More specifically, whereas cue image familiarity should be greater for images of studied names than for images of nonstudied names, access to information about the target may occur more often in the nonstudied than in the studied condition. In support of this assertion, the partial-identification rates (Table 2) were consistently higher in the nonstudied condition than in the studied condition across all three experiments. The likely reason for this is that targets that might otherwise have been partially accessed are more likely to be fully accessed after the target itself is primed through prior presentation at study (e.g., Cleary, 2006; Cleary \& Reyes, 2009; Rastle \& Burke, 1996). Priming through prior presentation increases the likelihood that a would-be near-accessible target will be fully accessible. Thus, the pool of studied targets contains, on average, more difficult-to-retrieve items for which priming did not lead to full access and for which partial access is therefore less likely. In contrast, the pool of nonstudied targets contains less-difficult-to-retrieve items for which near access or partial access is more likely.

Whereas the RWI condition may orient participants toward relying on cue familiarity, the FOK condition may orient participants toward relying on accessibility. Not only were participants more likely to partially access information about inaccessible targets in the nonstudied than in the studied conditions, but a trend in our study (which was statistically significant in Exp. 2a) was that partial access tended to be greatest in the nonstudied category of the FOK condition. This suggests that participants may have been trying harder to access information in the FOK condition than in the RWI condition.

It is important to note that we removed instances of correct partial identification of the targets from the ratings given to unidentified test images, which were the primary pool of data under consideration in the present study. Therefore, if accessibility indeed drove participants' ratings in the FOK condition, the type of access was likely either nonverbalizable partial information or idiosyncratic partial information (e.g., the participant recalled watching a movie at a friend's house in which that test image had been seen, but nothing specific regarding the name of the image). As Koriat (1993, 1995) has pointed out, participants do not necessarily have access to the correctness of the partial information that they access; thus, use of accessibility for making FOK judgments often leads people to be incorrect in their predictions. Thus, if participants are following a strategy whereby greater 
accessibility of any type of information at all in response to the test cue is attributed to the likelihood that the target name will be recognized later, they will often be wrong, such as in cases in which the accessed information is not actually diagnostic of how well the name will be recognized (as when one only recalls seeing the test image in a movie, but nothing specific about the name). Therefore, if we assume that the pool of nonstudied targets consists of items for which any type of partial information is going to be more accessible, and that participants are more likely to rely on accessibility in the FOK condition than in the RWI condition, this can explain why participants' ratings are higher for unidentified images of nonstudied target names than for unidentified images of studied target names in the FOK condition.

\section{Summary and conclusions}

In summary, RWI and FOK judgments led to different patterns of responding in response to the manipulation of the study status of inaccessible targets. Whereas retrospective recognition ratings for inaccessible targets were higher when targets were studied than when they were not studied (the RWI effect), the reverse occurred when participants rated their FOK judgments. We argue that participants are oriented toward relying on cue familiarity when the decision about an inaccessible target pertains to its prior occurrence in the experiment, and are oriented toward relying on accessibility when the decision pertains to its future remembrance.

Author note This work was funded by National Science Foundation Grant BCS-0638486 to A.M.C.

\section{References}

Benjamin, A. S. (2005). Response speeding mediates the contributions of cue familiarity and target retrievability to metamnemonic judgments. Psychonomic Bulletin \& Review, 12, 874-879. doi:10.3758/ BF03196779

Busey, T. A., Tunnicliff, J., Loftus, G. R., \& Loftus, E. F. (2000). Accounts of the confidence-accuracy relation in recognition memory. Psychonomic Bulletin \& Review, 7, 26-48. doi:10.3758/ BF03210724

Clark, S. E., \& Gronlund, S. D. (1996). Global matching models of recognition memory: How the models match the data. Psychonomic Bulletin \& Review, 3, 37-60. doi:10.3758/BF03210740

Cleary, A. M. (2004). Orthography, phonology, and meaning: Word features that give rise to feelings of familiarity in recognition. Psychonomic Bulletin \& Review, 11, 446-451. doi:10.3758/ BF03196593

Cleary, A. M. (2006). Relating familiarity-based recognition and the tipof-the-tongue phenomenon: Detecting a word's recency in the absence of access to the word. Memory \& Cognition, 34, 804-816.

Cleary, A. M., \& Greene, R. L. (2000). Recognition without identification. Journal of Experimental Psychology: Learning, Memory, and Cognition, 26, 1063-1069.
Cleary, A. M., \& Greene, R. L. (2001). Memory for unidentified items: Evidence for the use of letter information in familiarity processes. Memory \& Cognition, 29, 540-545.

Cleary, A. M., Langley, M. M., \& Seiler, K. R. (2004). Recognition without picture identification: Geons as components of the pictorial memory trace. Psychonomic Bulletin \& Review, 11, 903-908. doi:10.3758/BF03196719

Cleary, A. M., Morris, A. L., \& Langley, M. M. (2007). Recognition memory for novel stimuli: The structural regularity hypothesis. Journal of Experimental Psychology: Learning, Memory, and Cognition, 33, 379-393.

Cleary, A. M., \& Reyes, N. L. (2009). Scene recognition without identification. Acta Psychologica, 131, 53-62. doi:10.1016/ j.actpsy.2009.02.006

Cleary, A. M., Ryals, A. J., \& Nomi, J. (2009). Can déjà vu result from similarity to a prior experience? Support for the similarity hypothesis of déjà vu. Psychonomic Bulletin \& Review, 16, 1082-1088.

Cleary, A. M., \& Specker, L. E. (2007). Recognition without face identification. Memory \& Cognition, 35, 1610-1619.

Cleary, A. M., Winfield, M. M., \& Kostic, B. (2007). Auditory recognition without identification. Memory \& Cognition, 35, 18691877. doi:10.3758/BF03192921

Costermans, J., Lories, G., \& Ansay, C. (1992). Confidence level and feeling of knowing in question answering: The weight of inferential processes. Journal of Experimental Psychology: Learning, Memory, and Cognition, 18, 142-150.

Cree, G. S., McNorgan, C., \& McRae, K. (2006). Distinctive features hold a privileged status in the computation of word meaning: Implications for theories of semantic memory. Journal of Experimental Psychology: Learning, Memory, and Cognition, 32, 643-658.

Cree, G. S., \& McRae, K. (2003). Analyzing the factors underlying the structure and computation of the meaning of chipmunk, cherry, chisel, cheese and cello (and many other such concrete nouns). Journal of Experimental Psychology. General, 132, 163-201. doi:10.1037/0096-3445.132.2.163

Diana, R. A., Reder, L. M., Arndt, J., \& Park, H. (2006). Models of recognition: A review of arguments in favor of a dual-process account. Psychonomic Bulletin \& Review, 13, 1-21. doi:10.3758/ BF03193807

Dougherty, M. R., Scheck, P., Nelson, T. O., \& Narens, L. (2005). Using the past to predict the future. Memory \& Cognition, 33, 1096-1115.

Gruneberg, M. M., \& Monks, J. (1974). "Feeling of knowing" and cued recall. Acta Psychologica, 38, 257-265. doi:10.1016/00016918(74)90010-9

Hart, J. T. (1965). Memory and the feeling-of-knowing experience. Journal of Educational Psychology, 56, 208-216.

Hertzog, C., Dunlosky, J., \& Sinclair, S. M. (2010). Episodic feelingof-knowing resolution derives from the quality of original encoding. Memory \& Cognition, 38, 771-784. doi:10.3758/MC. 38.6.771

Izaute, M., Chambers, P., \& Larochelle, S. (2002). Feeling-of-knowing for proper names. Canadian Journal of Experimental Psychology, 56, 263-272. doi:10.1037/h0087402

Jacoby, L. L., \& Whitehouse, K. (1989). An illusion of memory: False recognition influenced by unconscious perception. Journal of Experimental Psychology. General, 118, 126-135. doi:10.1037/ 0096-3445.118.2.126

Koriat, A. (1993). How do we know that we know? The accessibility model of the feeling of knowing. Psychological Review, 100, 609-639. doi:10.1037/0033-295X.100.4.609

Koriat, A. (1995). Dissociating knowing and the feeling of knowing: Further evidence for the accessibility model. Journal of Experimental Psychology. General, 124, 311-333. doi:10.1037/00963445.124.3.311

Koriat, A. (2007). Metacognition and consciousness. In P. D. Zelazo, M. Moscovitch, \& E. Thompson (Eds.), Cambridge handbook of 
consciousness (pp. 289-325). New York, NY: Cambridge University Press.

Koriat, A., \& Levy-Sadot, R. (2001). The combined contributions of the cue-familiarity and accessibility heuristics to feelings of knowing. Journal of Experimental Psychology: Learning, Memory, and Cognition, 27, 34-53. doi:10.1037/0278-7393.27.1.34

Koriat, A., Levy-Sadot, R., Edry, E., \& de Marcas, S. (2003). What do we know about what we cannot remember? Accessing the semantic attributes of words that cannot be recalled. Journal of Experimental Psychology: Learning, Memory, and Cognition, 29, 1095-1105. doi:10.1037/0278-7393.29.6.1095

Kostic, B., \& Cleary, A. M. (2009). Song recognition without identification: When people cannot "name that tune" but can recognize it as familiar. Journal of Experimental Psychology. General, 138, 146-159. doi:10.1037/a0014584

Mandler, G. (1980). Recognizing: The judgment of previous occurrence. Psychological Review, 87, 252-271. doi:10.1037/0033295X.87.3.252

Mandler, G. (2008). Familiarity breeds attempts: A critical review of dual-process theories of recognition. Perspectives on Psychological Science, 3, 390-399. doi:10.1111/j.1745-6924.2008.00087.x

Maril, A., Simons, J. S., Weaver, J. J., \& Schacter, D. L. (2005). Graded recall success: An event-related fMRI comparison of tip of the tongue and feeling of knowing. NeuroImage, 24, 1130-1138.

Metcalfe, J., Schwartz, B. L., \& Joaquim, S. G. (1993). The cuefamiliarity heuristic in metacognition. Journal of Experimental Psychology: Learning, Memory, and Cognition, 19, 851-864. doi:10.1037/0278-7393.19.4.851

Mitchell, T. M., Shinkareva, S. V., Carlson, A., Chang, K. M., Malave, V. L., Mason, R. A., \& Just, M. A. (2008). Predicting human brain activity with the meanings of nouns. Science, 30, 1191-1195.

Nelson, T. O., Gerler, D., \& Narens, L. (1984). Accuracy of feeling of knowing judgments for predicting perceptual identification and relearning. Journal of Experimental Psychology. General, 113, $282-300$

Peynircioğlu, Z. F. (1990). A feeling-of-recognition without identification. Journal of Memory and Language, 29, 493-500. doi:10.1016/0749-596X(90)90068-B

Rastle, K. G., \& Burke, D. M. (1996). Priming the tip of the tongue: Effects of prior processing on word retrieval in young and older adults. Journal of Memory and Language, 35, 586-605. doi:10.1006/jmla.1996.0031

Reder, L. M., Nhouyvanisvong, A., Schunn, C. D., Ayers, M. S., Angstadt, P., \& Hiraki, K. (2000). A mechanistic account of the mirror effect for word frequency: A computational model of remember-know judgments in a continuous recognition paradigm. Journal of Experimental Psychology: Learning, Memory, and Cognition, 26, 294-320. doi:10.1037/0278-7393.26.2.294

Reder, L. M., \& Ritter, F. E. (1992). What determines initial feeling of knowing? Familiarity with question terms, not with the answer. Journal of Experimental Psychology: Learning, Memory, and Cognition, 18, 435-451.

Ryals, A. J., \& Cleary, A. M. (2012). The recognition without cued recall phenomenon: Support for a feature-matching theory over a partial recollection account. Journal of Memory and Language. doi:10.1016/j.jml.2012.01.002

Schacter, D. L. (1983). Feeling of knowing in episodic memory. Journal of Experimental Psychology: Learning, Memory, and Cognition, 9, 39-54. doi:10.1037/0278-7393.9.1.39

Schwartz, B. L. (2008). Working memory load differentially affects tip-of-the-tongue states and feeling-of-knowing judgments. Memory \& Cognition, 36, 9-19.

Schwartz, B. L., \& Metcalfe, J. (1992). Cue familiarity but not target retrievability enhances feeling-of-knowing judgments. Journal of Experimental Psychology: Learning, Memory, and Cognition, 18, 1074-1083.

Shimamura, A. P., Landwehr, R. F., \& Nelson, T. O. (1981). FACTRETRIEVAL: A program for assessing someone's recall of generalinformation facts, feeling-of-knowing judgments for nonrecalled facts, and recognition of nonrecalled facts. Behavior Research Methods \& Instrumentation, 13, 691-692.

Shimamura, A. P., \& Squire, L. R. (1986). Memory and metamemory: A study of the feeling-of-knowing phenomenon in amnesic patients. Journal of Experimental Psychology: Learning, Memory, and Cognition, 12, 452-460. doi:10.1037/0278-7393. 12.3 .452

Tversky, A., \& Kahneman, D. (1974). Judgment under uncertainty: Heuristics and biases. Science, 185, 1124-1131. doi:10.1126/ science.185.4157.1124

Wright, D. B. (1996). Measuring the feeling of knowing: Comment on Schraw. Applied Cognitive Psychology, 10, 261-268.

Yaniv, I., \& Meyer, D. E. (1987). Activation and metacognition of inaccessible stored information: Potential bases for incubation effects in problem solving. Journal of Experimental Psychology: Learning, Memory, and Cognition, 13, 187-205.

Yonelinas, A. P. (2002). The nature of recollection and familiarity: A review of 30 years of research. Journal of Memory and Language, 46, 441-517. doi:10.1006/jmla.2002.2864 